\title{
Article
}

\section{Post-AGB Discs from Common-Envelope Evolution}

\author{
Robert G. Izzard $1,2, *(1)$ and Adam S. Jermyn ${ }^{2}(\mathbb{D}$ \\ 1 Department of Physics, University of Surrey, Guildford, Surrey GU2 7XH, UK \\ 2 Institute of Astronomy, University of Cambridge, Madingley Road, Cambridge CB3 OHA, UK; \\ adamjermyn@gmail.com \\ * Correspondence: r.izzard@surrey.ac.uk
}

Received: 26 July 2018; Accepted: 4 September 2018; Published: 11 September 2018

\begin{abstract}
Post-asymptotic giant branch (post-AGB) stars with discs are all binaries. Many of these binaries have orbital periods between 100 and 1000 days so cannot have avoided mass transfer between the AGB star and its companion, likely through a common-envelope type interaction. We report on preliminary results of our project to model circumbinary discs around post-AGB stars using our binary population synthesis code binary_c. We combine a simple analytic thin-disc model with binary stellar evolution to estimate the impact of the disc on the binary, and vice versa, fast enough that we can model stellar population and hence explore the rather uncertain parameter space involved with disc formation. We find that, provided the discs form with sufficient mass and angular momentum, and have an inner edge that is relatively close to the binary, they can both prolong the life of their parent post-AGB star and pump the eccentricity of orbits of their inner binaries.
\end{abstract}

Keywords: binary stars; post-AGB; discs

\section{Introduction}

About half the stars exceeding the mass of the Sun are binaries, and of these many interact during their lifetime [1]. Many key astrophysical phenomena occur in binary systems, such as thermonuclear novae, X-ray bursts, type Ia supernovae and merging compact objects as detected by gravitational waves. Despite their importance to fundamental astrophysics, many aspects of binary evolution remain poorly understood.

The evolution of binary stars differs from that of single stars mostly because of mass transfer. In binaries wide enough that one star becomes a giant, yet short enough that the giant cannot fit inside the binary, mass transfer begins when the radius of the giant star exceeds its Roche radius. Because the giant is convective, and likely significantly more massive than its companion, mass transfer is unstable and accelerates. This leads to a common envelope forming around the stars. Drag between the stars and the envelope cause the orbit to decay and, if enough energy is transferred to the envelope, the envelope is ejected [2]. Stars on the giant branch or asymptotic giant branch (GB or AGB stars) are stripped almost down to their cores, leaving a post-(A)GB binary.

During the common envelope process, tides are expected to be highly efficient and hence the binary that emerges is expected to have little, if any, eccentricity $(e \approx 0)$. Yet, post-AGB binaries are often highly eccentric, up to $e=0.6$. The source of this eccentricity is unknown but is probably related to a similar phenomena observed in the barium stars which are thought to have involved mass transfer from an AGB star [3,4].

Many post-AGB stars have discs, and all those with discs are in binary systems [5]. Investigations into the link between these discs and their stellar systems' peculiar eccentricity, based on eccentricity pumping by Lindblad resonances, suggest that the discs can cause the post-AGB systems' eccentricity if they are sufficiently massive and live for long enough [6]. Recent ALMA observations, e.g., of 
IRAS 08544-4431, show that post-AGB discs are mostly Keplerian, have masses of about $10^{-2} \mathrm{M}_{\odot}$, outer diameters of $10^{16} \mathrm{~cm} \approx 10^{5} \mathrm{R}_{\odot}$, angular momenta similar to their parent binary systems (around $10^{52} \mathrm{~g} \mathrm{~cm}^{2} \mathrm{~s}^{-1}$ ), and both slow mass loss from the outer part of the disc and inflow at its inner edge at a rate of about $10^{-7} \mathrm{M}_{\odot}$ year $^{-1}$ [7].

In this work we combine a fast, analytic model of circumbinary discs with a synthetic binary stellar-evolution code to estimate the number of post-(A)GB discs and their properties. We include mass loss from the disc caused by illumination from the central star, ram-stripping by the interstellar medium, and include a viscous-timescale flow onto the central binary. The disc extracts angular momentum from its central binary star system. Resonances excited in the disc pump the central binary's eccentricity. While our results are preliminary, they show that eccentricity pumping is efficient in some systems, and the discs may live a considerable time.

\section{Circumbinary Disc Model}

We assume that circular, Keplerian discs form when a common envelope is ejected and some small fraction of the envelope mass, $f_{M} \ll 1$, is left behind as a disc containing a fraction, $f_{J} \ll 1$, of the envelope's angular momentum. The disc thus has mass $M_{\text {disc }}$ and angular momentum $J_{\text {disc }}$. We assume our discs are thin such that $H / R<1$, where $H(R)$ is the scale height at a radius $R$, and have viscous timescales that are short compared to their lifetimes such that they spread instantaneously [8]. Given that observed discs are mostly cool and neutral (i.e., $T \lesssim 1000 \mathrm{~K}$ in most of the disc), we fix the opacity to $\kappa=0.01 \mathrm{~m}^{2} \mathrm{~g}^{-1}$ and assume an $\alpha$-viscosity model with $\alpha=10^{-3}$.

Given the above simplifications, we write the heat-balance equation in the disc as,

$$
\sigma T^{4}=\mathcal{A}+\mathcal{B}(1+\mathcal{C})
$$

where $T=T(R)$ is the temperature in the disc mid-plane (cf. [9] where these terms are derived in detail). The $\sigma T^{4}$ term is the heating by the post-(A)GB star, which is balanced in equilibrium by visocus heating $(\mathcal{A})$ and re-radiation $(\mathcal{B}$ and $\mathcal{C})$. We neglect the impact of mass changes on the heat balance because observed discs have low mass-loss rates $\left(\lesssim 10^{-7} \mathrm{M}_{\odot}\right.$ year $\left.^{-1}\right)$. The terms $\mathcal{A}, \mathcal{B}$ and $\mathcal{C}$ are functions of radius, such that we can rewrite Equation (1) as,

$$
T^{4}=a \Sigma^{2} T R^{-3 / 2}+b T^{1 / 2} R^{-3 / 2}+c R^{-3},
$$

where $\Sigma=\Sigma(R)$ is the surface density at radius $R$. At a given radius $R$ we then choose the largest term in the right hand side of Equation (2) and set the other terms to zero, allowing a simple solution for $T(R)$ everywhere in the disc (cf. [10]). Errors in $T^{4}$ are up to a factor of 3, hence errors in $T$ are at most $3^{1 / 4} \approx 30 \%$ and typically much less, which is good enough for our purposes.

To verify that we can neglect mass changes, note that the temperature, $T_{\text {acc }}$, associated with accretion at a rate $\dot{M}$ at radius $R$ and binary mass $M_{\text {binary }}[9]$,

$$
\sigma T_{\text {acc }}^{4}=\frac{G M_{\text {binary }} \dot{M}}{2 \pi R^{3}}
$$

where the gravitational mass is dominated by the binary. Evaluating this yields,

$$
T_{\text {acc }}(R)=290 \mathrm{~K}\left(\frac{\dot{M}}{10^{-7} \mathrm{M}_{\odot} \text { year }^{-1}}\right)^{1 / 4}\left(\frac{M_{\text {binary }}}{\mathrm{M}_{\odot}}\right)^{1 / 4}\left(\frac{R}{10^{2} \mathrm{R}_{\odot}}\right)^{-3 / 4} .
$$

At the inner edges of our discs $R \approx 10^{2} \mathrm{R}_{\odot}$ so $T_{\text {acc }}$ is a factor of several smaller than the typical $1000 \mathrm{~K}$ temperatures we find. The outer edges, at $R \approx 10^{5} \mathrm{R}_{\odot}$, have $T_{\text {acc }} \approx 2 \mathrm{~K}$ which both cooler than the disc and the Cosmic Microwave background so this term can be safely ignored.

We next scale the density and outer radius so that the integrals of mass and angular momentum throughout the disc match $M_{\text {disc }}$ and $J_{\text {disc }}$ respectively, while the inner radius $R_{\text {in }}$ is fixed by the torque 
on the disc caused by the inner binary which we take from [11] with a multiplier of $10^{-3}$. Thus, given three constraints- $M_{\text {disc }} J_{\text {disc }}$ and the inner binary torque-we know $T(R)$ throughout the disc. From this we construct any other required physical quantities, and can integrate these to calculate, for example, the luminosity of the disc. With our chosen torque prescription, our discs' inner radii are typically around twice the orbital separation, as assumed in other works (e.g., [12]).

We treat mass loss from the circumbinary disc as a slow phenomenon. At the inner edge of the disc we include mass inflow onto the central binary at the local viscous rate. At the outer edge we strip material when its pressure is less than that of the interstellar medium, assumed to be $3000 \mathrm{~K} / k_{\mathrm{B}}$ where $k_{\mathrm{B}}$ is the Boltzmann constant. Irradiation by the post-(A)GB star, particularly in X-rays, causes mass loss and we model this with the prescription of [13]. During most of the disc's lifetime, the viscous inflow is most important, but X-ray losses dominate when the star transitions to become a hot, young white dwarf and these losses quickly evaporate the disc. Our neglect of mass loss in Equation (1) is incorrect in this brief phase but because the disc is terminated very rapidly, on timescales of years, such systems will be rarely observed.

The evolution of the binary stars is calculated using binary_c [14-17]. Common envelopes are ejected with the formalism of [18] using an efficiency $\alpha_{\mathrm{CE}}=1$ to match observed post-AGB systems with periods between 100 and about 1000 days which are observed to have circumbinary discs. The envelope binding energy parameter, $\lambda_{\mathrm{CE}}$, is fitted to the models of [19], and $10 \%$ of the envelope's recombination energy is used to aid ejection. Because typically $\lambda_{\mathrm{CE}} \gg 1$ during the AGB, such envelopes are nearly unbound and common envelope ejection is efficient with only modest orbital shrinkage.First giant branch stars have $\lambda \lesssim 1$ because they are more tightly bound, so their orbits shrink significantly. We also assume that stars exit the common envelope with a small eccentricity, $e=10^{-5}$, to which we apply Lindblad resonance pumping [6]. If $\alpha_{\mathrm{CE}}$ is less than 1.0, as suggested by e.g., [20-22], then more recombination energy can be included to prevent orbital shrinkage. We do not pretend to better understand common envelope evolution with our simple model.

At the end of the common envelope phase our treatment differs from [18] in that we keep a thin envelope on the (A)GB star such that it just fills its Roche lobe when the envelope is ejected. Typically this is a $10^{-2}-10^{-3} \mathrm{M}_{\odot}$ hydrogen-rich envelope which keeps the star cool $(\sim 5000 \mathrm{~K})$ during the post-(A)GB phase relative to the white dwarf $\left(\gtrsim 10^{4} \mathrm{~K}\right)$ it will become. The star continues its nuclear burning which reduces the mass of the envelope. Accretion from the circumbinary disc replenishes the shell and extends the lifetime of the post-(A)GB phase, as we show in the following section.

\section{Example System}

As an example binary star system we choose an initial primary mass $M_{1}=1.5 \mathrm{M}_{\odot}$, initial secondary mass $M_{2}=0.9 \mathrm{M}_{\odot}$, initial separation $a=800 \mathrm{R}_{\odot}$ and metallicity $Z=0.02$. The separation is chosen such that Roche-lobe overflow is initiated just after the primary starts thermally pulsing on the AGB. Common envelope evolution follows with $\alpha_{\mathrm{CE}} \lambda_{\mathrm{CE}}=2.1$ so the orbit shrinks to $195 \mathrm{R}_{\odot}$. The primary is then a $0.577 \mathrm{M}_{\odot}$ post-AGB star, with an envelope mass of $7 \times 10^{-3} \mathrm{M}_{\odot}$ and a $0.95 \mathrm{M}_{\odot}$ main-sequence dwarf companion. The $0.05 \mathrm{M}_{\odot}$ accreted on to the secondary is from the wind of the AGB star prior to common envelope evolution. The orbital period is then about 255 days, typical of post-AGB binaries with circumbinary discs. We model circular orbits but our model is equally applicable to initially mildly eccentric binaries. Tides are expected to be efficient as the primary ascends the AGB and will quickly circularize the system. In the following discussion, and the figures, times are measured from the moment the common envelope is ejected.

A circumbinary disc is formed with $f_{M}=0.02$ and $f_{J}=0.107$, giving $M_{\text {disc }}=0.012 \mathrm{M}_{\odot}$ and $J_{\text {disc }}=10^{52} \mathrm{~g} \mathrm{~cm}^{2} \mathrm{~s}^{-1}$. Both $f_{M}$ and $f_{J}$ are chosen to give us a disc with mass and angular momentum similar that of IRAS 08544-4431 [7] and other post-AGB systems with circumbinary discs (e.g., the Red Rectangle). As the disc evolves, it feeds off the angular momentum of the inner binary, but the total angular momentum gained during its lifetime is small. Mass flows through the inner edge onto the binary at between $10^{-7}$ (initially) and $10^{-8} \mathrm{M}_{\odot}$ year ${ }^{-1}$, although this does not significantly alter 
the evolution of the disc. Rather, X-ray driven mass loss, caused by the post-AGB star increasing in temperature at approximately constant luminosity, leads to sudden termination of the disc at $6.5 \times 10^{4}$ years, as shown in Figure 1 .

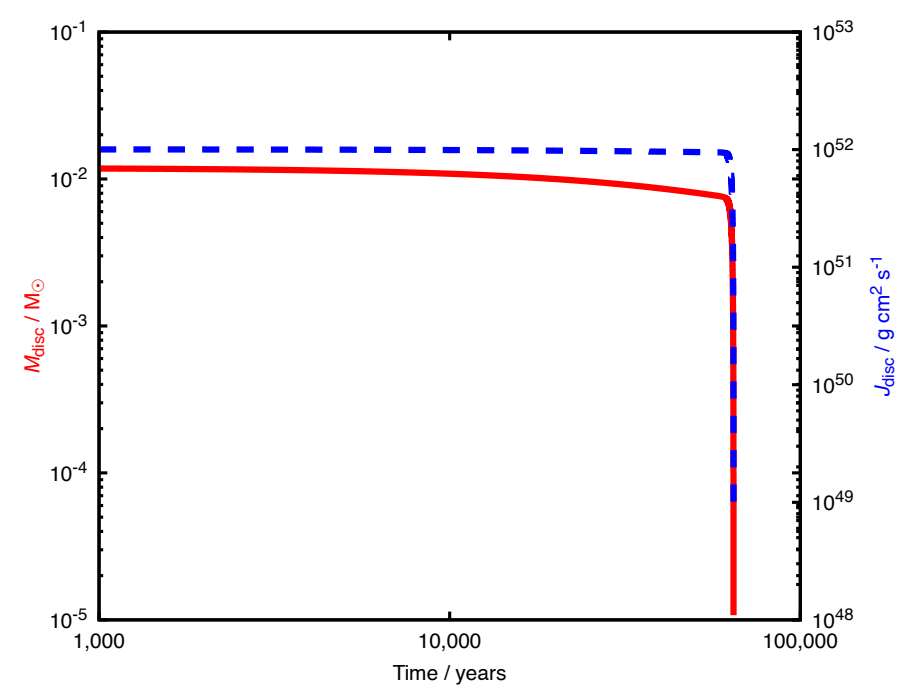

Figure 1. Mass (left axis, red solid line) and angular momentum (right axis, blue dashed line) evolution in our example circumbinary disc system. During most of the evolution of the disc, its mass changes slowly $\left(\sim 10^{-8} \mathrm{M}_{\odot}\right.$ year $\left.^{-1}\right)$ because of viscous flow through its inner edge onto the inner binary system. As the post-AGB star in the binary heats up, its X-ray flux increases until it drives sufficient wind that the disc is quickly evaporated after about $6.5 \times 10^{4}$ years where a time of 0 years is when the common envelope is ejected.

The inner edge of the disc is at $317 \mathrm{R}_{\odot}$, well outside the inner binary orbit, while the outer edge is at $4.7 \times 10^{4} \mathrm{R}_{\odot}$. The former is set by the applied torque, while the latter is set by the disc angular momentum. Until the disc is evaporated, neither the inner nor outer radius changes significantly. Figure 2 shows the evolution of said radii.

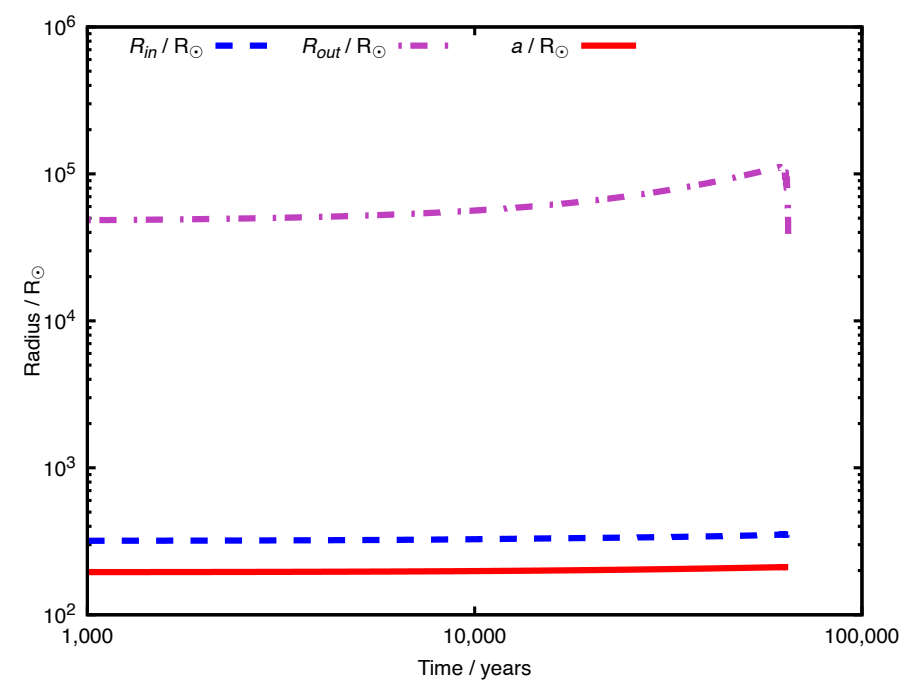

Figure 2. Evolution of the inner and outer radii, $R_{\text {in }}$ and $R_{\text {out }}$ respectively, and the binary orbital separation $a$, in our example circumbinary disc system. Time is measured from the moment of common envelope ejection. 
The eccentricity of the inner binary system is pumped to about 0.25 by the time the disc is evaporated. This is similar to the eccentricity observed in post-AGB systems, and it is certainly non-zero.

Mass accreted onto the post-AGB star from the inner edge of the disc replenishes its hydrogen-rich envelope, thus cooling the star and prolonging its lifetime. Because our post-AGB star has a core mass of only $0.57 \mathrm{M}_{\odot}$, its nuclear burning rate is similar to the disc's viscous accretion rate. The stellar wind mass loss rate is less than $10^{-11} \mathrm{M}_{\odot}$ year ${ }^{-1}$ because we apply the rate of [23] in our ignorance of the mechanism of post-AGB wind loss. To test how long accretion extends the post-AGB, we evolved an identical example system but with the inner-edge viscous inflow disabled. Figure 3 shows that the post-AGB star in the system with accretion lives for an extra $3 \times 10^{4}$ years, an approximate doubling of the its post-AGB lifetime. The extra lifetime of such systems may explain why they do not show residual nebulosity from envelope ejection. Observed planetary nebulae, which may be ejected common envelopes, have dynamical timescales of about $10^{4}$ years. By the time the post-AGB star is hot enough to ionize such envelopes they are likely too diffuse to be observed as planetary nebulae [24].

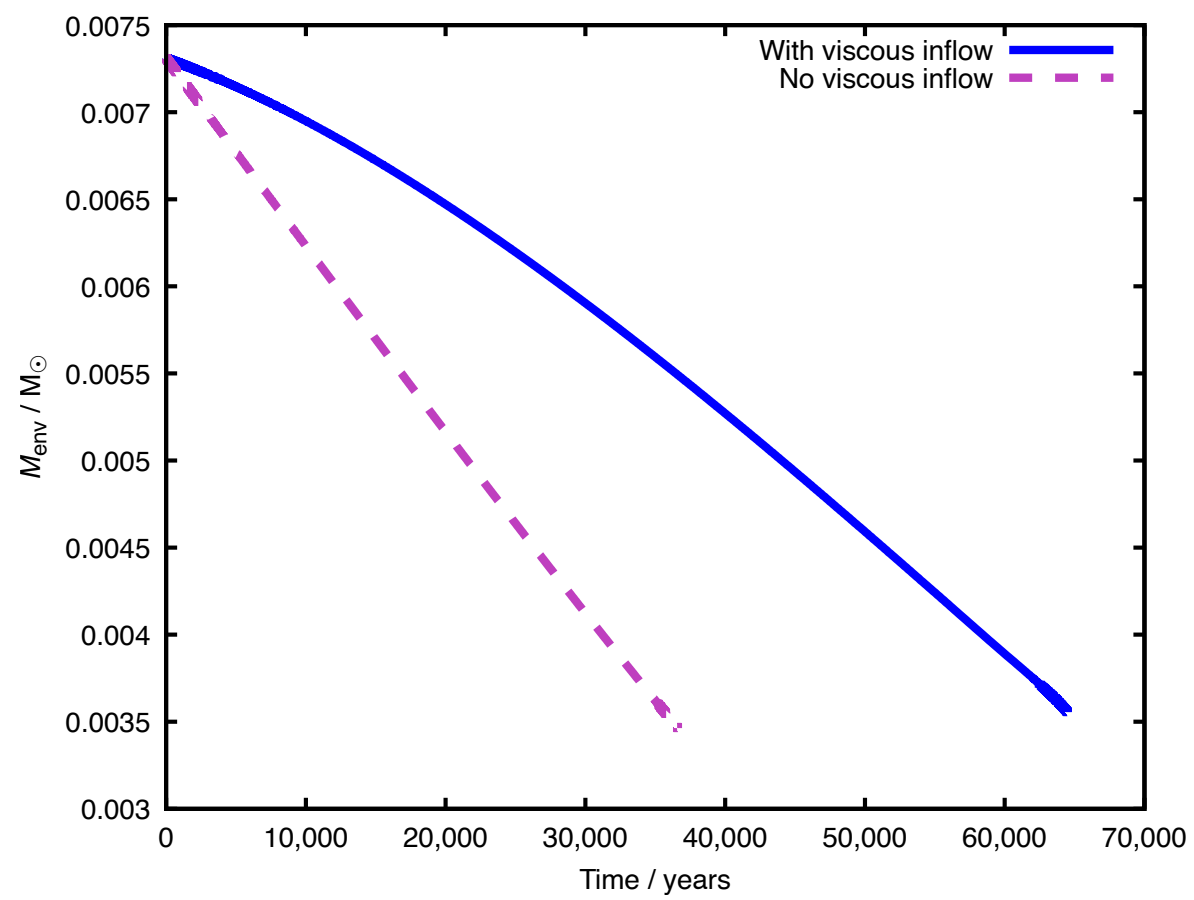

Figure 3. Post-AGB envelope mass vs. time since common envelope ejection. The blue, solid line is our example system with mass inflow from the inner edge onto the inner binary, while the magenta, dashed line is without. Mass flowing onto the post-AGB star replenishes its hydrogen envelope thus, for a while, keeps it relatively cool, limits its X-ray flux and prevents it from evaporating the disc. In this case, its lifetime is extended from about 35,000 years to 65,000 years. In our example system, post-AGB wind mass loss is negligible.

\section{Stellar Populations and Improved Physics}

Our model is simple yet it contains the essential physics of circumbinary discs around post-(A)GB stars. It is also fast enough that we can evolve a typical binary system containing a circumbinary disc in just a few seconds of CPU time. Speed is a an essential requirement of binary population synthesis studies because the parameter space is large. We can explore the consequence of the initial mass and angular momentum of our discs through the input parameters $f_{M}$ and $f_{J}$, and we can estimate the effect of changing uncertain input physics, e.g., a stronger or weaker X-ray wind or binary torque.

We can also model post-first giant branch (helium core) systems and post-early-AGB systems. From an observational point of view, these differ in that their stellar evolution is truncated at an earlier stage than in post-thermally-pulsing-AGB, hence they are dimmer. These systems also overflow their 
Roche lobes at shorter periods and have more bound envelopes, so their orbits and resultant discs are more compact. Our assumption of constant opacity likely breaks at this point, although our assumed instantaneous viscous spreading of the disc is certainly valid. We are working on improving the model to take this into account.

Our discs are low in mass relative to their stars and we never put more than $10 \%$ of the common envelope mass in the disc. The example system we report in Section 3 has a Toomre $Q$ parameter of about $10^{8}$ [25] so is not gravitationally unstable. However, this does not preclude the formation of rocks in the disc, after all we know the discs contain small grains which emit in the infra red. In our models we expect a small number of discs to form in systems that exit the red giant branch just before helium ignition. These systems will contain sdB stars rather than white dwarfs, thus stay relatively cool. The relatively low X-ray flux from sdB stars means their discs are not evaporated quickly, at least not by means modelled here, so long-lived discs and the formation of so-called debris, i.e., rocks, in them discs seems quite likely.

The formation of second-generation planets in our discs seems not to be favoured. Our discs live for less than $10^{5}$ years, too short for planet formation in the canonical sense, and our discs are quite warm, hotter than $1000 \mathrm{~K}$, near the inner edge where they are densest. That said, circumbinary discs have material concentrated in their orbital plane of the system for some time, so if even minor planets survive the common-envelope phase, they could accrete material from the disc. The consequences for the disc may be that it does not survive but this is currently beyond the scope of our model.

Our discs do succeed in pumping the eccentricity of their inner binary, in the case of our example system up to 0.25 . In part this is because our discs are, by design, quite massive (about $0.01 \mathrm{M}_{\odot}$ ), but this is to match observed discs such as IRAS 08544-4431, so is reasonable. Circumbinary discs seem to be good candidates to explain the eccentricities of at least some post-mass-transfer objects such as barium stars. We have not yet tested wind mass transfer as a mechanism for disc formation, but this is an obvious extension to our work and likely contributes to the observed population of post-(A)GB binaries.

Author Contributions: Conceptualization, data curation, funding acquisition, project administration, resources, software, supervision and writing-original draft; R.G.I. Formal analysis, investigation, methodology, validation, visualization and writing-review and editing; R.G.I. and A.S.J.

Funding: This research was funded by the Science and Technology Facilities Council under grant number ST/L003910/1.

Acknowledgments: RGI thanks the STFC for funding his Rutherford fellowship under grant ST/L003910/1, Churchill College, Cambridge for his fellowship and access to their library. Hello to Jason Isaacs. ASJ thanks the UK Marshall Commission for financial support.

Conflicts of Interest: The authors declare no conflict of interest.

\section{References}

1. De Marco, O.; Izzard, R.G. Dawes Review 6: The Impact of Companions on Stellar Evolution. Publ. Astron. Soc. Aust. 2017, 34, e001. [CrossRef]

2. Ivanova, N.; Justham, S.; Chen, X.; De Marco, O.; Fryer, C.L.; Gaburov, E.; Ge, H.; Glebbeek, E.; Han, Z.; Li, X.D.; et al. Common envelope evolution: Where we stand and how we can move forward. Astron. Astrophys. Rev. 2013, 21, 59. [CrossRef]

3. Jorissen, A.; Van Eck, S.; Mayor, M.; Udry, S. Insights into the formation of barium and Tc-poor S stars from an extended sample of orbital elements. Astron. Astrophys. 1998, 332, 877-903.

4. Karakas, A.I.; Tout, C.A.; Lattanzio, J.C. The eccentricities of the barium stars. Mon. Not. R. Astron. Soc. 2000, 316, 689-698. [CrossRef]

5. Bujarrabal, V.; Alcolea, J.; Van Winckel, H.; Santander-García, M.; Castro-Carrizo, A. Extended rotating disks around post-AGB stars. Astron. Astrophys. 2013, 557, A104. [CrossRef]

6. Dermine, T.; Izzard, R.G.; Jorissen, A.; Van Winckel, H. Eccentricity-pumping in post-AGB stars with circumbinary discs. Astron. Astrophys. 2013, 551, A50. [CrossRef] 
7. Bujarrabal, V.; Castro-Carrizo, A.; Winckel, H.V.; Alcolea, J.; Contreras, C.S.; Santander-García, M.; Hillen, M. High-resolution observations of IRAS 08544-4431. Detection of a disk orbiting a post-AGB star and of a slow disk wind. Astron. Astrophys. 2018, 614, A58. [CrossRef] [PubMed]

8. Lynden-Bell, D.; Pringle, J.E. The evolution of viscous discs and the origin of the nebular variables. Mon. Not. R. Astron. Soc. 1974, 168, 603-637. [CrossRef]

9. Perets, H.B.; Kenyon, S.J. Wind-accretion Disks in Wide Binaries, Second-generation Protoplanetary Disks, and Accretion onto White Dwarfs. Astrophys. J. 2013, 764, 169. [CrossRef]

10. Haiman, Z.; Kocsis, B.; Menou, K. The Population of Viscosity- and Gravitational Wave-driven Supermassive Black Hole Binaries Among Luminous Active Galactic Nuclei. Astrophys. J. 2009, 700, 1952-1969. [CrossRef]

11. Armitage, P.J.; Natarajan, P. Accretion during the Merger of Supermassive Black Holes. Astrophys. J. Lett. 2002, 567, L9-L12. [CrossRef]

12. Rafikov, R.R. On the Eccentricity Excitation in Post-main-sequence Binaries. Astrophys. J. 2016, 830, 8. [CrossRef]

13. Owen, J.E.; Clarke, C.J.; Ercolano, B. On the theory of disc photoevaporation. Mon. Not. R. Astron. Soc. 2012, 422, 1880-1901. [CrossRef]

14. Izzard, R.G.; Tout, C.A.; Karakas, A.I.; Pols, O.R. A new synthetic model for asymptotic giant branch stars. Mon. Not. R. Astron. Soc. 2004, 350, 407-426. [CrossRef]

15. Izzard, R.G.; Dray, L.M.; Karakas, A.I.; Lugaro, M.; Tout, C.A. Population nucleosynthesis in single and binary stars. I. Model. Astron. Astrophys. 2006, 460, 565-572. [CrossRef]

16. Izzard, R.G.; Glebbeek, E.; Stancliffe, R.J.; Pols, O.R. Population synthesis of binary carbon-enhanced metal-poor stars. Astron. Astrophys. 2009, 508, 1359-1374. [CrossRef]

17. Izzard, R.G.; Preece, H.; Jofre, P.; Halabi, G.M.; Masseron, T.; Tout, C.A. Binary stars in the Galactic thick disc. Mon. Not. R. Astron. Soc. 2018, 473, 2984-2999. [CrossRef]

18. Hurley, J.R.; Tout, C.A.; Pols, O.R. Evolution of binary stars and the effect of tides on binary populations. Mon. Not. R. Astron. Soc. 2002, 329, 897-928. [CrossRef]

19. Tauris, T.M.; Dewi, J.D.M. Research Note On the binding energy parameter of common envelope evolution. Dependency on the definition of the stellar core boundary during spiral-in. Astron. Astrophys. 2001, 369, 170-173. [CrossRef]

20. Zorotovic, M.; Schreiber, M.R.; Gänsicke, B.T.; Nebot Gómez-Morán, A. Post-common-envelope binaries from SDSS. IX: Constraining the common-envelope efficiency. Astron. Astrophys. 2010, 520, A86. [CrossRef]

21. De Marco, O.; Passy, J.C.; Moe, M.; Herwig, F.; Mac Low, M.M.; Paxton, B. On the $\alpha$ formalism for the common envelope interaction. Mon. Not. R. Astron. Soc. 2011, 411, 2277-2292. [CrossRef]

22. Davis, P.J.; Kolb, U.; Knigge, C. Is the common envelope ejection efficiency a function of the binary parameters? Mon. Not. R. Astron. Soc. 2012, 419, 287-303, [CrossRef]

23. Vassiliadis, E.; Wood, P.R. Evolution of low- and intermediate-mass stars to the end of the asymptotic giant branch with mass loss. Astrophys. J. 1993, 413, 641-657. [CrossRef]

24. Keller, D.; Izzard, R.G.; Stanghellini, L.; Lau, H.B. Planetary nebulae: Binarity, composition and morphology. Unpublished work, 2018.

25. Toomre, A. On the gravitational stability of a disk of stars. Astrophys. J. 1964, 139, 1217-1238. [CrossRef]

(C) 2018 by the authors. Licensee MDPI, Basel, Switzerland. This article is an open access article distributed under the terms and conditions of the Creative Commons Attribution (CC BY) license (http://creativecommons.org/licenses/by/4.0/). 